\title{
A NOTE ON THE SENSITIVITY ANALYSIS FOR THE SYMPLECTIC QR FACTORIZATION
}

\author{
HANYU Li AND PENG LV
}

Abstract. In this note, the rigorous perturbation bounds for $R$ factor of the implicit Bunch form of the symplectic QR factorization under normwise perturbation are derived by using the block matrix-vector equation approach, the technique of Lyapunov majorant function, and the Banach fixed point principle. These bounds are tighter than the one in [ $\mathrm{Li}$ et al. Linear Multilinear Algebra, 63, (2015), 78-96] and can be regarded as the rigorous versions of the optimal firstorder perturbation bounds in [Li et al. J. Franklin Inst., 353, 5 (2016), 1186-1205].

Mathematics subject classification (2010): 15A23, 15A45.

Keywords and phrases: Symplectic QR factorization, rigorous perturbation bound, Lyapunov majorant function, Banach fixed point principle.

\section{REFERENCES}

[1] R. BhatiA, Matrix factorizations and their perturbations, Linear Algebra Appl. 197, (1994), 245 276.

[2] A. BunSE-Gerstner, Matrix factorizations for symplectic QR-like methods, Linear Algebra Appl. 83, (1986), 49-77.

[3] A. Bunse-Gerstner And V. Mehrmann, A symplectic QR-like algorithm for the solution of the real algebraic Riccati equation, IEEE Trans. Automat. Control 31, 12 (1986), 1104-1113.

[4] X. W. ChAng, On the sensitivity of the SR decomposition, Linear Algebra Appl. 282, (1998), $297-$ 310.

[5] R. A. Horn And C. R. Johnson, Topics in Matrix Analysis, Cambridge University Press, Cambridge, 1991.

[6] M. Konstantinov, D. Gu, V. Mehrmann and P. PetKov, Perturbation Theory for Matrix Equations, Elsevier, Amsterdam, 2003.

[7] R. H. Koning, H. Neudecker And T. WAnsBeek, Block Kronecker products and the vecb operator, Linear Algebra Appl. 149, (1991), 165-184.

[8] D. KRESSNER, Block algorithms for orthogonal symplectic factorizations, BIT 43, (2003), 775-790.

[9] H. Y. LI AND Y. M. WEI, Improved rigorous perturbation bounds for the $L U$ and QR factorizations, Linear Algebra Appl. 22, (2015), 1115-1130.

[10] H. Y. LI, H. YANG AND H. ShaO, Perturbation analysis for the symplectic QR factorization, Linear Multilinear Algebra 63, (2015), 78-96.

[11] H. Y. LI, Y. M. WEI AND Y. F. YANG, New rigorous perturbation bounds for the Cholesky-like factorization of skew-symmetric matrix, Linear Algebra Appl. 491, (2016), 83-100.

[12] W. LI, Z. J. XIE AND S. W. Vong, Sensitivity analysis for the symplectic QR factorization, J. Franklin Inst. 353, 5 (2016), 1186-1205.

[13] S. SINGER AND S. SINGER, Rounding error and perturbation bounds for the symplectic QR factorization, Linear Algebra Appl. 358, (2003), 255-279.

[14] G. M. Stewart And J. G. Sun, Matrix Perturbation Theory, Academic Press, Boston, 1990.

[15] D. S. WATKins And L. ElSner, Self-similar flows, Linear Algebra Appl. 110, (1988), 213-242. 